\title{
PROPER MOTIONS IN THE BULGE: LOOKING THROUGH PLAUT'S LOW EXTINCTION WINDOW
}

\author{
R.A. MÉNDEZ ${ }^{1}$, R.M. RICH ${ }^{2}$, W.F. VAN ALTENA ${ }^{3}$, \\ T.M. GIRARD ${ }^{3}$, S. VAN DEN BERGH ${ }^{4}$ AND S.R. MAJEWSKI ${ }^{5}$ \\ ${ }^{1}$ European Southern Observatory \\ ${ }^{2}$ Columbia University, Astronomy Department \\ ${ }^{3}$ Yale University, Astronomy Department \\ ${ }^{4}$ Dominion Astrophysical Observatory \\ ${ }^{5}$ University of Virginia, Department of Astronomy
}

\section{A Survey for Proper-motions}

We are conducting the deepest and largest photographic proper-motion survey ever undertaken of the Galactic bulge. Our first-epoch plate material (from 1972-3) goes deep enough $\left(V_{l i m} \sim 22\right)$ to reach below the bulge mainsequence turnoff. These plates cover an area of approximately $25^{\prime} \times 25^{\prime}$ of the bulge in the low-extinction $\left(A_{v} \sim 0.8 \mathrm{mag}\right)$ Plaut field at $\mathrm{l}=0^{\circ}, \mathrm{b}=$ $-8^{\circ}$, approximately $1 \mathrm{kpc}$ south of the nucleus. This is the point at which the transition between bulge and halo populations likely occurs and is, therefore, an excellent location to study the interface between the dense metal-rich bulge and the metal-poor halo.

In order to study the formation of the bulge and its chemical evolution one would like to measure proper-motions, radial velocities, and abundances for members of that stellar population, in a way similar to that of the seminal effort by Eggen, Lynden-Bell \& Sandage in 1962, which addressed the oldest stellar population in our Galaxy.

\section{Survey Description and Preliminary Results}

Our project is based on a unique sample of twenty photographic plates of a Galactic bulge field on the minor-axis at $b=-8^{\circ}$ obtained by Sidney van den Bergh in 1972-3 using the Kitt Peak 84-inch and the Palomar 200inch telescopes. The 100-inch telescope at Las Campanas has been used to 
obtain thirteen second epoch plates in 1993; deep intermediate epoch plates of this field (1979) were obtained by Jeremy Mould also at Las Campanas.

The plates were digitized using the the Yale PDS 2020G laser interferometer/microdensitometer measuring machine. Analysis of the Yale-PDS microdensitometer data routinely yields star centroids to $1 / 20$ of a pixel (20 mas). A star measured on five plates in each color will have its position known at least 2-3 times better than this, and over the 21 yr baseline we expect errors no larger than 0.5 mas $\mathrm{yr}^{-1}$ in each color. This corresponds to approximately $20 \mathrm{~km} \mathrm{~s}^{-1}$ at the distance of the Galactic Center. This is comparable to Spaenhauer et al. (1992), and matches the accuracy with which radial velocities in our spectroscopic follow-up will be measured.

Preliminary results, based on only three first epoch and three last-epoch plates spanning 21 years (Méndez et al. 1996) indicate that it is possible to obtain proper-motions with errors less than 1 mas $\mathrm{yr}^{-1}$ for a about 5,000 stars down to $\mathrm{V}=18$, without color restriction. For the subsample with errors less than 1 mas $\mathrm{yr}^{-1}$ we derive proper-motion dispersions in the direction of Galactic longitude and latitude of $3.378 \pm 0.033$ mas $^{-1}$ and $2.778 \pm 0.028$ mas $\mathrm{yr}^{-1}$ respectively. These dispersions agree with those derived by Spaenhauer et al. (1992) in Baade's window.

We expect to measure CCD photometry in the B, V, and I passbands, and proper-motions for an unbiased sample of approximately 30,000 stars in our minor-axis field. We hope to further obtain radial velocities and low resolution abundances for about 5,000 stars. A large, unbiased sample is important because much of the outcome depends on dividing the data into subsamples as a function of abundance or kinematics. Radial velocities from our spectroscopic survey will be extremely important as a complement to the proper-motions to confirm the presence of a stellar bar in the Bulge.

\section{References}

Méndez, R.A., Rich, R.M., van Altena, W.F., Girard, T.M., van den Bergh, S., \& Majewski, S.R., 1996, in "The Galactic Center," 4th ESO/CTIO Workshop, Astron.Soc.Pacific Conference Series, 102, R. Gredel, ed. (ASP, San Francisco) p. 345

Spaenhauer, A., Jones, B.F., Whitford, E., 1992, Astron.J., 103, 297 